\title{
Half-Time Image Reconstruction in Thermoacoustic Tomography
}

\author{
Mark A. Anastasio*, Jin Zhang, Xiaochuan Pan, Yu Zou, Geng Ku, and Lihong V. Wang
}

\begin{abstract}
Thermoacoustic tomography (TAT) is an emerging imaging technique with great potential for a wide range of biomedical imaging applications. In this paper, we propose and investigate reconstruction approaches for TAT that are based on the half-time reflectivity tomography paradigm. We reveal that half-time reconstruction approaches permit for the explicit control of statistically complementary information that can result in the optimal reduction of image variances. We also show that half-time reconstruction approaches can mitigate image artifacts due to heterogeneous acoustic properties of an object. Reconstructed images and numerical results produced from simulated and experimental TAT measurement data are employed to demonstrate these effects.

Index Terms-Image reconstruction, photoacoustic tomography, reflectivity tomography, spherical Radon transform, thermoacoustic tomography.
\end{abstract}

\section{INTRODUCTION}

$\mathbf{T}$ HERMOACOUSTIC tomography (TAT) is an emerging imaging technique with great potential for a wide range of biomedical imaging applications [1]-[4]. In TAT, a short electromagnetic pulse (e.g., microwave or laser) is used to irradiate a biological tissue. When the electromagnetic pulse is absorbed by the tissue, a thermoacoustic effect results in the emission of acoustic signals that can be measured by use of a wide-band ultrasonic transducer. The objective of TAT is to produce an image that represents a map of the spatially variant electromagnetic absorption properties of the tissue. Such an image may be of great value because a strong correlation exists between electromagnetic absorption in biological tissue and the pathological condition of the tissue [5], [6].

Although prototype TAT scanners have been built and investigated recently, there remains a great need for the development of accurate and robust tomographic reconstruction algorithms. Exact [3] and approximate [1], [7] reconstruction algorithms have been developed for TAT employing spherical scanning apertures. Equivalently, image reconstruction can be accomplished through transformation of the TAT data function into a reflectivity tomography data function [8]-[10], followed

Manuscript received May 26, 2004; revised October 8, 2004. The Associate Editor responsible for coordinating the review of this paper and recommending its publication was C. R. Crawford. Asterisk indicates corresponding author.

$*$ M. A. Anastasio is with the Department of Biomedical Engineering, Illinois Institute of Technology, 10 W. 32nd St., E1-116, Chicago, IL 60616 USA (e-mail: anastasio@iit.edu).

J. Zhang is with the Department of Biomedical Engineering, Illinois Institute of Technology, Chicago, IL 60616 USA.

X. Pan and Y. Zou are with the Department of Radiology, The University of Chicago, Chicago, IL 60637 USA.

G. Ku and L. V. Wang are with the Department of Biomedical Engineering, Texas A\&M University, College Station, TX 77843USA.

Digital Object Identifier 10.1109/TMI.2004.839682 by application of a reflectivity tomography reconstruction algorithm. However, a common feature of these reconstruction approaches is that they require complete knowledge of the data functions. Moreover, they do not provide for the explicit exploitation of data redundancies that are inherent in the tomographic measurements.

Previously we have investigated the so-called half-time reconstruction problem of reflectivity tomography. Using a layerstripping type of procedure [11] and concepts from microlocal analysis, we demonstrated [12], [13] that an image could be reconstructed uniquely and stably from measurement data, acquired at all source-receiver locations on the scanning aperture, that were truncated at delay time $T_{\text {trun }} \geq T_{\text {half }}$. Here, $T_{\text {half }}$ represents twice the time it takes for the probing wavefield to propagate from the source to the center of the scanning aperture. In the language of tomography, at each view angle (i.e., source-receiver position), half-time image reconstruction requires knowledge of the set of integrals of the object function over spherical surfaces that have radii less than or equal to the radius of the scanning aperture. In this sense, the half-time reconstruction problem of reflectivity tomography is analogous to the half-detector problem [14] of X-ray tomography with a parallel-beam tomography.

In this paper, we propose and investigate reconstruction approaches for TAT that are based on the half-time reflectivity tomography paradigm. We reveal that half-time reconstruction approaches permit for the explicit control of statistically complementary information that can result in the optimal reduction of reconstructed image variances. We also show that half-time reconstruction approaches can mitigate image artifacts due to heterogeneous acoustic properties of an object. Reconstructed images and numerical results are produced from simulated and experimental two-dimensional (2-D) TAT measurement data to demonstrate these effects.

\section{REVIEW OF TAT IMAGING MODEL}

Consider an electromagnetic pulse with time dependence $\delta(t)$ that is used to irradiate a tissue sample. When the electromagnetic pulse is absorbed by the tissue, a thermoacoustic effect results in the generation of a pressure wavefield $p(\vec{r}, t)$ that can be measured by use of a wide-band ultrasonic transducer that is located on the surface of a spherical measurement aperture $\Omega_{0}$ that encloses the sample (see Fig. 1). In the 2-D case, the measurement aperture $\Omega_{0}$ corresponds to a circle. Assuming that the object to be imaged has homogeneous acoustic properties (but spatially varying electromagnetic absorption 


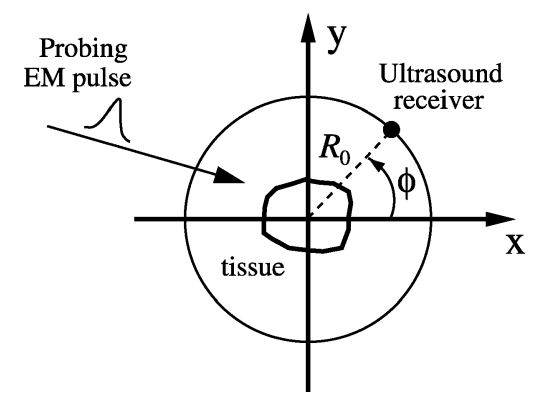

Fig. 1. TAT measurement configuration.

properties), the pressure wavefield $p\left(\vec{r}_{0}, t\right)$ that is measured ${ }^{1}$ as a function of time at location $\vec{r}_{0} \in \Omega_{0}$ can be expresses as [3]

$$
p\left(\vec{r}_{0}, t_{0}\right)=\eta \int d^{3} \vec{r} A(\vec{r}) \frac{d}{d t_{0}} \frac{\delta\left(t_{0}-\frac{\left|\vec{r}_{0}-\vec{r}\right|}{c_{0}}\right)}{4 \pi\left|\vec{r}_{0}-\vec{r}\right|}
$$

where $A(\vec{r})$ denotes the spatial energy absorption function that resides inside a sphere of radius $R_{f}, c_{0}$ is the (constant) velocity of sound in the object and background medium, and the constant $\eta$ represents the isobaric volume expansion coefficient divided by the specific heat of the (acoustically) homogeneous medium. The reconstruction problem of TAT is to invert (1) for determination of $A(\vec{r})$ from knowledge of $p\left(\vec{r}_{0}, t_{0}\right)$ for $\vec{r}_{0} \in \Omega_{0}$ and $t_{0} \in[0, \infty)$. Assuming three-dimensional (3-D) scanning apertures, inversion formulae have been derived [3], [7] that can accomplish this.

A mathematical relationship between the TAT data function and the spherical Radon transform can be derived readily. Specifically, by acting $\int_{0}^{t} d t_{0}$ on both sides of (1), one can show that

$$
g\left(\vec{r}_{0}, \bar{t}\right) \equiv \frac{4 \pi}{\eta} t \int_{0}^{t} d t_{0} p\left(\vec{r}_{0}, t_{0}\right)=\int d^{3} \vec{r} A(\vec{r}) \delta\left(\bar{t}-\left|\vec{r}_{0}-\vec{r}\right|\right)
$$

where $\bar{t} \equiv c_{0} t$ and $g\left(\vec{r}_{0}, \bar{t}\right)$ can be interpreted as a spherical Radon transform of $A(\vec{r})$. Equation (2) has the same mathematical form as the imaging model in reflectivity tomography [8], [9]. Consequently, image reconstruction in TAT can be accomplished by inverting the spherical Radon transform in (2) via a reflectivity tomography reconstruction algorithm.

\section{A. Half-Time TAT Data Functions}

Consider half-time data functions $g^{(1)}\left(\vec{r}_{0} ; \bar{t}\right)$ and $g^{(2)}\left(\vec{r}_{0} ; \bar{t}\right)$ that are defined $\forall \vec{r}_{0} \in \Omega_{0}$ as

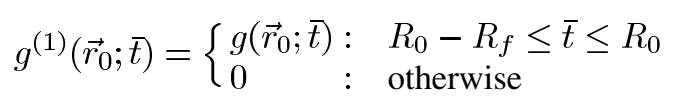

and

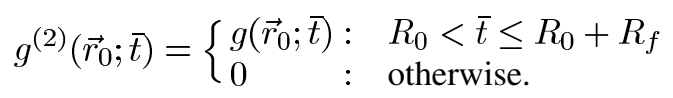

${ }^{1}$ In practice, the function $p\left(\vec{r}_{0}, t_{0}\right)$ is deconvolved by the impulse response of the receiving transducer and the finite width of the probing electromagnetic pulse.
Existing reflectivity tomography reconstruction algorithms [8]-[10] assume that the data are complete (untruncated), and therefore will generally produce distorted images when applied directly to $g^{(1)}\left(\vec{r}_{0} ; \bar{t}\right)$ or $g^{(2)}\left(\vec{r}_{0} ; \bar{t}\right)$. Previously, we have employed a layer-stripping procedure [12], [13] to explicitly identify redundant information in the (untruncated) data function $g\left(\vec{r}_{0} ; \bar{t}\right)$ and demonstrated that $A(\vec{r})$ is determined uniquely from knowledge of the half-time data functions $g^{(1)}\left(\vec{r}_{0} ; \bar{t}\right)$ or $g^{(2)}\left(\vec{r}_{0} ; \bar{t}\right)$. A similar result has been derived recently by Finch et al. [10] using a different mathematical approach. To date, exact and explicit inversion formulae for reconstruction of $A(\vec{r})$ from knowledge of the half-time data functions $g^{(1)}\left(\vec{r}_{0} ; \bar{t}\right)$ or $g^{(2)}\left(\vec{r}_{0} ; \bar{t}\right)$ remain undiscovered. However, we have demonstrated that accurate images can be reconstructed stably from knowledge of the half-time data functions by use of iterative reconstruction algorithms [12], [13]. Another iterative reconstruction approach for TAT is described in [15].

\section{B. Discrete TAT Imaging Model}

In practice, the detected pressure wavefield is discretized temporally and measured at a finite number of receiver locations. Let $p\left[\vec{r}_{0}^{k}, t_{l}\right]$ denote the discretized pressure signal, where $t_{l} \equiv$ $l \Delta t, l=0,2, \cdots, N_{s}-1$ describes the $N_{s}$ temporal sampling points with sampling interval $\Delta t$ and the vectors $\vec{r}_{0}^{k}, k=$ $1,2,3, \cdots, N_{r}$ represent the $N_{r}$ receiver locations on the aperture $\Omega_{0}$. A discrete approximation of (2) is given by

$$
\begin{aligned}
g\left[\vec{r}_{0}^{k}, \bar{t}_{l}\right] & =\frac{4 \pi}{\eta} t_{l} \sum_{m=0}^{l} \Delta t p\left[\vec{r}_{0}^{k}, t_{m}\right] \\
& \approx \int d^{3} \vec{r} A(\vec{r}) \delta\left(\bar{t}_{l}-\left|\vec{r}_{0}^{k}-\vec{r}\right|\right)
\end{aligned}
$$

where $\bar{t}_{l} \equiv c_{0} t_{l}$ and the notation $g[\cdot, \cdot]$ denotes a sampled version of the continuous function $g(\cdot, \cdot)$. Similarly, the sampled versions of the half-time data functions $g^{(1)}\left(\vec{r}_{0} ; \bar{t}\right)$ and $g^{(2)}\left(\vec{r}_{0} ; \bar{t}\right)$ will be denoted by $g^{(1)}\left[\vec{r}_{0}^{k}, \bar{t}_{l}\right]$ and $g^{(2)}\left[\vec{r}_{0}^{k}, \bar{t}_{l}\right]$, respectively.

\section{EXPloitATION OF STATISTICALLy COMPLEMENTARY DATA}

In this section, we describe how half-time reconstruction approaches, along with knowledge of the (second-order) data noise statistics, can be employed to form statistically optimal reconstruction procedures for TAT.

\section{A. Noise Model}

With consideration of data noise, the detected pressure wavefield can be described as

$$
\boldsymbol{p}\left[\vec{r}_{0}^{k}, t_{l}\right]=p\left[\vec{r}_{0}^{k}, t_{l}\right]+\boldsymbol{n}\left[\vec{r}_{0}^{k}, t_{l}\right]
$$

where $\boldsymbol{n}\left[\vec{r}_{0}^{k}, t_{l}\right]$ is an additive noise component that models detector noise and/or stochastic variations in the pressure wavefield. Here and elsewhere, boldface and normal typefaces denote a stochastic quantity and its mean, respectively. The operators $\operatorname{Var}\{\cdot\}$ and Covar $\{\cdot, \cdot\}$ will denote the variance and covariance, respectively, of the associated stochastic quantities. According 
to (5) and (6), $\boldsymbol{g}\left[\vec{r}_{0}^{k}, \bar{t}_{l}\right]$ is also a stochastic quantity with covariance

$$
\begin{aligned}
\operatorname{Covar}\{ & \left.\boldsymbol{g}\left[\vec{r}_{0}^{k}, \bar{t}_{l}\right], \boldsymbol{g}\left[\vec{r}_{0}^{k^{\prime}}, \bar{t}_{l^{\prime}}\right]\right\}=\left(\frac{4 \pi \Delta t}{\eta}\right)^{2} t_{l} t_{l^{\prime}} \\
& \times \sum_{m=0}^{l} \sum_{m^{\prime}=0}^{l^{\prime}} \operatorname{Covar}\left\{\boldsymbol{n}\left[\vec{r}_{0}^{k}, t_{m}\right], \boldsymbol{n}\left[\vec{r}_{0}^{k^{\prime}}, t_{m^{\prime}}\right]\right\} .
\end{aligned}
$$

Equation (7) describes how the second-order statistics of the data noise determine the second-order statistics of the (spherical Radon transform) data function $\boldsymbol{g}\left[\vec{r}_{0}^{k}, \bar{t}_{l}\right]$.

It is often reasonable to assume that $\boldsymbol{n}\left[\vec{r}_{0}^{k}, t_{l}\right]$ is an uncorrelated stochastic process [16] that is described by

$$
\operatorname{Covar}\left\{\boldsymbol{n}\left[\vec{r}_{0}^{k}, t_{m}\right], \boldsymbol{n}\left[\vec{r}_{0}^{k^{\prime}}, t_{m^{\prime}}\right]\right\}=\sigma_{n}^{2}\left[\vec{r}_{0}^{k}, t_{m}\right] \delta_{k, k^{\prime}} \delta_{m, m^{\prime}}
$$

where $\sigma_{n}^{2}\left[\vec{r}_{0}^{k}, t_{m}\right] \equiv \operatorname{Var}\left\{\boldsymbol{n}\left[\vec{r}_{0}^{k}, t_{m}\right]\right\}$ and $\delta_{k, k^{\prime}}$ denotes the Kronecker delta function. Substitution from (8) into (7) results in

$$
\begin{aligned}
\operatorname{Covar}\left\{\boldsymbol{g}\left[\vec{r}_{0}^{k}, \bar{t}_{l}\right], \boldsymbol{g}\left[\vec{r}_{0}^{k^{\prime}}, \bar{t}_{l^{\prime}}\right]\right\} \\
=\delta_{k, k^{\prime}}\left(\frac{4 \pi \Delta t}{\eta}\right)^{2} t_{l} t_{l^{\prime}} \sum_{m=0}^{l_{<}} \sigma_{n}^{2}\left[\vec{r}_{0}^{k}, t_{m}\right]
\end{aligned}
$$

where $l_{<}$is the smaller of $l$ and $l^{\prime}$. In the case of stationary noise with $\sigma_{n}^{2}\left[\vec{r}_{0}^{k}, t_{m}\right]=\sigma_{n}^{2}$, (9) reduces to

$\operatorname{Covar}\left\{\boldsymbol{g}\left[\vec{r}_{0}^{k}, \bar{t}_{l}\right], \boldsymbol{g}\left[\vec{r}_{0}^{k^{\prime}}, \bar{t}_{l^{\prime}}\right]\right\}=\delta_{k, k^{\prime}}\left(\frac{4 \pi \Delta t}{\eta}\right)^{2} t_{l} t_{l^{\prime}} \sigma_{n}^{2}\left(l_{<}+1\right)$.

Because $\sigma_{n}^{2}\left[\vec{r}_{0}^{k}, t_{m}\right]>0$, (9) and/or (10) indicate that the noise in $\boldsymbol{g}\left[\vec{r}_{0}^{k}, \bar{t}_{l}\right]$ is correlated with respect to the $\bar{t}_{l}$-coordinate and $\operatorname{Var}\left\{g\left[\vec{r}_{0}^{k}, \bar{t}_{l}\right]\right\}$ is a monotonically increasing function of $\bar{t}_{l}$. Therefore values of $\boldsymbol{g}\left[\vec{r}_{0}^{k}, \bar{t}_{l}\right]$ that correspond to large $\bar{t}_{l}$ may be corrupted significantly by noise.

\section{B. Data Function Signal-to-Noise Ratio}

For the noise models discussed in Section III-A, the magnitude of the noise in $\boldsymbol{g}^{(1)}\left[\vec{r}_{0}, \vec{t}\right]$ will be smaller than in $\boldsymbol{g}^{(2)}\left[\vec{r}_{0}, \vec{t}\right]$. Depending on the structure of the object, this can result in $\boldsymbol{g}^{(1)}\left[\vec{r}_{0}, \vec{t}\right]$ having a larger signal-to-noise ratio (SNR) than $\boldsymbol{g}^{(2)}\left[\vec{r}_{0}, \vec{t}\right]$. As an example of this effect, we can calculate analytically the SNR of the noisy circular Radon transform data function $\boldsymbol{g}\left[\vec{r}_{0}, \vec{t}\right]$ for the 2-D case where $A(\vec{r})$ is comprised of uniform disks and $\Omega_{0}$ represents a circle with radius $R_{0}$. (A similar analysis can be performed readily for the 3-D case where $A(\vec{r})$ is comprised of uniform spheres, but it provides qualitatively similar conclusions.)

Consider that $A(\vec{r})$ is a uniform disk with unit amplitude that is centered at the origin and has a radius $R_{f}$. For a given $\vec{r}_{0}^{k} \in$ $\Omega_{0}$, from elementary geometry one finds that

$$
\begin{aligned}
\mathrm{SNR} & \equiv \frac{g\left[\vec{r}_{0}^{k} ; \bar{t}_{l}\right]}{\sqrt{\operatorname{Var}\left\{\boldsymbol{g}\left[\vec{r}_{0}^{k}, \bar{t}_{l}\right]\right\}}} \\
& =\frac{2 \bar{t}_{l}}{\sqrt{\operatorname{Var}\left\{\boldsymbol{g}\left[\vec{r}_{0}^{k}, \bar{t}_{l}\right]\right\}}} \cos ^{-1}\left[\frac{R_{0}^{2}-R_{f}^{2}+\bar{t}_{l}^{2}}{2 \bar{t}_{l} R_{0}}\right]
\end{aligned}
$$

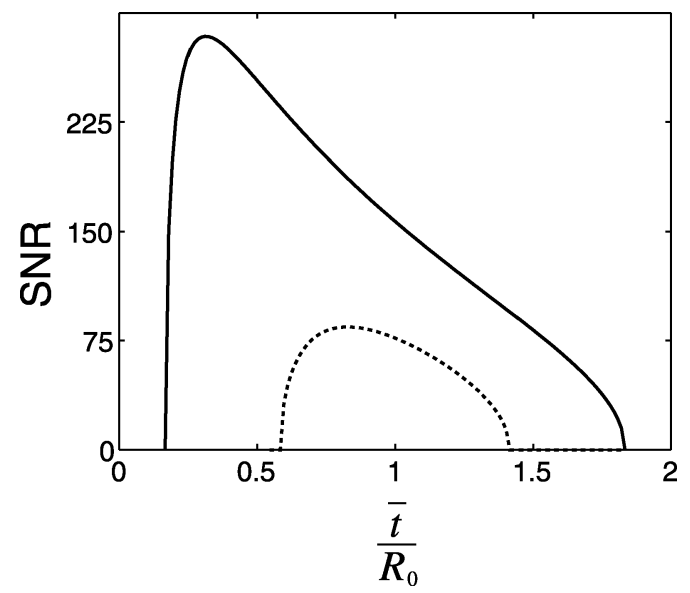

Fig. 2. A plot of (12) for the cases $R_{f}=1.0$ (solid line) and $R_{f}=0.5$ (dashed line). The parameters $R_{0}=1.2$ and $D_{0}=1$ were employed.

where recall that $R_{0}$ is the radius of the scanning aperture. For the noise model corresponding to (10) one obtains

$$
\mathrm{SNR}=\frac{D_{0}}{\sqrt{t}_{l}} \cos ^{-1}\left[\frac{R_{0}^{2}-R_{f}^{2}+\bar{t}_{l}^{2}}{2 \bar{t}_{l} R_{0}}\right]
$$

where $D_{0} \equiv \eta \sqrt{c_{0}} / 2 \pi \sigma_{n} \sqrt{\Delta t}$. A plot of (12) as a function of $\bar{t}_{l}$ for $R_{f} / R_{0}=0.83$ (solid line) and $R_{f} / R_{0}=0.42$ (dashed line) are given in Fig. 2. These plots show that the data function $\boldsymbol{g}^{(1)}\left[\vec{r}_{0}^{k}, \bar{t}_{l}\right]$ (that corresponds to $\left.\left(\bar{t}_{l} / R_{0}\right) \in[0,1]\right)$ possesses larger SNR values than does the data function $\boldsymbol{g}^{(2)}\left[\vec{r}_{0}^{k}, \bar{t}_{l}\right]$ (that corresponds to $\left.\bar{t}_{l} / R_{0} \in(1,2]\right)$. Note that this effect becomes more pronounced as $\left(R_{f} / R_{0}\right) \rightarrow 1$. This is significant because it is highly desirable to have $R_{f} / R_{0} \approx 1$ in compact TAT scanners. In Section VI, we demonstrate numerically that, for objects of practical interest, images reconstructed from $\boldsymbol{g}^{(1)}\left[\vec{r}_{0}^{k}, \bar{t}_{l}\right]$ have significantly lower variances than those reconstructed from $g^{(2)}\left[\vec{r}_{0}^{k}, \bar{t}_{l}\right]$.

\section{Explicit Control of Image Variance}

As described in Section II-A, either of the half-time data functions $g^{(1)}\left[\vec{r}_{0}^{k}, \bar{t}_{l}\right]$ or $g^{(2)}\left[\vec{r}_{0}^{k}, \bar{t}_{l}\right]$ can be employed for reconstruction of the image $A(\vec{r})$. This indicates that the (complete) data function $g\left[\vec{r}_{0}^{k}, \bar{t}_{l}\right]$ contains a twofold redundancy. In the presence of data noise, the images reconstructed from $g^{(1)}\left[\vec{r}_{0}^{k}, \bar{t}_{l}\right]$ and $g^{(2)}\left[\vec{r}_{0}^{k}, \bar{t}_{l}\right]$ will not be identical in general. One way to reconstruct an image $A(\vec{r})$ that has an optimally reduced variance without introducing a bias is to identify and exploit statistically complementary information that is contained in $\boldsymbol{g}^{(1)}\left[\vec{r}_{0}^{k}, \bar{t}_{l}\right]$ and $\boldsymbol{g}^{(2)}\left[\vec{r}_{0}^{k}, \bar{t}_{l}\right]$. When the data functions are noiseless and consistent, this is tantamount to identifying redundancies in $g^{(1)}\left[\vec{r}_{0}^{k}, \bar{t}_{l}\right]$ and $g^{(2)}\left[\vec{r}_{0}^{k}, \bar{t}_{l}\right]$. Unfortunately, simple point-to-point mappings that relate values of $g^{(1)}\left[\vec{r}_{0}^{k}, \bar{t}_{l}\right]$ and $g^{(2)}\left[\vec{r}_{0}^{k}, \bar{t}_{l}\right]$ in data space do not exist [11]. This is unlike the conventional Radon transform case for which data redundancies can be identified readily by use of obvious symmetry properties. Although data redundancies are not identified easily in the data space, as described next, we can utilize the half-time reconstruction paradigm to exploit statistically complementary information in $\boldsymbol{g}^{(1)}\left[\vec{r}_{0}^{k}, \bar{t}_{l}\right]$ and $\boldsymbol{g}^{(2)}\left[\vec{r}_{0}^{k}, \bar{t}_{l}\right]$ and thereby control the statistical properties of the reconstructed image. 
Let $\boldsymbol{A}^{(1)}(\vec{r}), \boldsymbol{A}^{(2)}(\vec{r})$, and $\boldsymbol{A}(\vec{r})$ denote estimates of $A(\vec{r})$ that are reconstructed from $\boldsymbol{g}^{(1)}\left[\vec{r}_{0}^{k}, \bar{t}_{l}\right], \boldsymbol{g}^{(2)}\left[\vec{r}_{0}^{k}, \bar{t}_{l}\right]$, and $\boldsymbol{g}\left[\vec{r}_{0}^{k}, \bar{t}_{l}\right]$, respectively. For example, in Section VI we reconstruct such images by use of a nonlinear iterative reconstruction algorithm. In general, these images will contain statistically complementary information that can be utilized to form an estimate of $A(\vec{r})$ that has a reduced variance. For example, we can form the estimate

$$
\boldsymbol{A}_{i, j}^{\omega}(\vec{r})=\omega_{i, j}(\vec{r}) \boldsymbol{B}_{i}(\vec{r})+\left[1-\omega_{i, j}(\vec{r})\right] \boldsymbol{B}_{j}(\vec{r})
$$

where $\boldsymbol{B}_{i}(\vec{r})$ and $\boldsymbol{B}_{j}(\vec{r})$ represent any two distinct images in the three element set

$$
\left\{\boldsymbol{B}_{1}(\vec{r})=\boldsymbol{A}^{(1)}(\vec{r}), \boldsymbol{B}_{2}(\vec{r})=\boldsymbol{A}^{(2)}(\vec{r}), \boldsymbol{B}_{3}(\vec{r})=\boldsymbol{A}(\vec{r})\right\}
$$

and $\omega_{i, j}(\vec{r})$ is a real-valued combination coefficient. For a given pair of images to combine [i.e, for a given $\boldsymbol{B}_{i}(\vec{r})$ and $\boldsymbol{B}_{j}(\vec{r})$ ], (13) represents an infinite family of estimators for obtaining $\boldsymbol{A}_{i, j}^{\omega}(\vec{r})$ that is indexed by the choice of $\omega_{i, j}$. Because $\boldsymbol{B}_{i}(\vec{r})$ and $B_{j}(\vec{r})$ are chosen from a set of three possible images, (13) describes three infinite families of such estimators.

From (13) the variance of $\boldsymbol{A}_{i, j}^{\omega}(\vec{r})$ can be expressed as

$$
\begin{aligned}
\operatorname{Var}\left\{\boldsymbol{A}_{i, j}^{\omega}(\vec{r})\right\}= & \omega_{i, j}(\vec{r})^{2} \operatorname{Var}\left\{\boldsymbol{B}_{i}(\vec{r})\right\}+\left[1-\omega_{i, j}(\vec{r})\right]^{2} \\
& \times \operatorname{Var}\left\{\boldsymbol{B}_{j}(\vec{r})\right\}+2 \omega_{i, j}^{2}(\vec{r})\left[1-\omega_{i, j}(\vec{r})\right] \\
& \times \operatorname{Covar}\left\{\boldsymbol{B}_{i}(\vec{r}), \boldsymbol{B}_{j}(\vec{r})\right\} .
\end{aligned}
$$

It is clear, therefore, that different choices for $\omega_{i, j}(\vec{r})$ will produce estimators $\boldsymbol{A}_{i, j}^{\omega}(\vec{r})$ that have different variances. To obtain $A_{i, j}^{\omega}(\vec{r})$ that has an optimally reduced variance, we can choose $\omega_{i, j}(\vec{r})$ such that

$$
\left.\frac{\partial \operatorname{Var}\left\{\boldsymbol{A}_{i, j}^{\omega}(\vec{r})\right\}}{\partial \omega_{i, j}}\right|_{\omega_{i, j}=\omega_{i, j}^{o p t}}=0
$$

which defines an optimal combination coefficient $\omega_{i, j}^{o p t}$ that minimizes (14). Substitution from (14) into (15) yields

$\omega_{i, j}^{o p t}=\frac{\operatorname{Var}\left\{\boldsymbol{B}_{j}(\vec{r})\right\}-\operatorname{Covar}\left\{\boldsymbol{B}_{i}(\vec{r}), \boldsymbol{B}_{j}(\vec{r})\right\}}{\operatorname{Var}\left\{\boldsymbol{B}_{i}(\vec{r})\right\}+\operatorname{Var}\left\{\boldsymbol{B}_{j}(\vec{r})\right\}-2 \operatorname{Covar}\left\{\boldsymbol{B}_{i}(\vec{r}), \boldsymbol{B}_{j}(\vec{r})\right\}}$.

Therefore, from knowledge of the (joint) second-order statistical properties of $\boldsymbol{B}_{i}(\vec{r})$ and $\boldsymbol{B}_{j}(\vec{r})$, an estimate $\boldsymbol{A}_{i, j}^{\omega}(\vec{r})$ can be formed that has the minimum variance obtainable by the corresponding family of estimators described by (13). The identification of the family of estimators that can produce an estimate of $A(\vec{r})$ that has the smallest variance depends on the second-order statistics of the data function $\boldsymbol{g}\left[\vec{r}_{0}^{k}, \bar{t}_{l}\right]$ (and hence the second-order statistics of $\boldsymbol{g}^{(1)}\left[\vec{r}_{0}^{k}, \bar{t}_{l}\right]$ and $\left.\boldsymbol{g}^{(2)}\left[\vec{r}_{0}^{k}, \bar{t}_{l}\right]\right)$ and the choice of the algorithms that are used for reconstruction of the images $\boldsymbol{A}^{(1)}(\vec{r}), \boldsymbol{A}^{(2)}(\vec{r})$, and $\boldsymbol{A}(\vec{r})$. In Section V, we employ several noise models and a given reconstruction algorithm to investigate numerically the families of estimators described by (13).

\section{Mitigation of Artifacts From Acoustic INHOMOGENEITIES}

In this section, we describe how half-time reconstruction approaches can mitigate artifacts that arise from heterogeneities in the acoustic properties of an object.

\section{A. Acoustic Heterogeneities in TAT}

It is customary in TAT to assume that the object is acoustically homogeneous. As described by (1) and (2), under this assumption the TAT image reconstruction problem can be solved by inverting a spherical Radon transform. In many applications of TAT, including imaging of the female breast [4], the object of interest may not possess a constant acoustic speed. In these cases, (1) is an approximation and the images obtained by inverting (2) can contain distortions and artifacts.

In [4], the effects of acoustic heterogeneities in TAT breast imaging were studied. It was demonstrated that multi-path interference in breast TAT was negligible, but phase distortions caused by time-of-flight (TOF) variations could be problematic. It is reasonable to assume that similar conclusions apply to other imaging applications involving objects that possess only small variations in acoustic properties. To account for acoustic heterogeneities, the forward model in (1) can be generalized as

$$
\hat{p}\left(\vec{r}_{0}, t_{0}\right)=\eta \int d^{3} \vec{r} A(\vec{r}) \frac{d}{d t_{0}} \frac{\delta\left[t_{0}-t_{f}\left(\vec{r}, \vec{r}_{0}\right)\right]}{4 \pi\left|\vec{r}_{0}-\vec{r}\right|},
$$

where $t_{f}\left(\vec{r}, \vec{r}_{0}\right)$ describes the TOF from point $\vec{r}$ to $\vec{r}_{0}$. As described in [4], $t_{f}\left(\vec{r}, \vec{r}_{0}\right)$ can be approximated accurately as

$$
t_{f}\left(\vec{r}, \vec{r}_{0}\right)=\int_{\vec{r}^{\prime} \in L\left(\vec{r}, \vec{r}_{0}\right)} d^{3} \vec{r}^{\prime} \frac{1}{c\left(\vec{r}^{\prime}\right)}
$$

where $c(\vec{r})$ is the local acoustic speed and the set $L\left(\vec{r}, \vec{r}_{0}\right)$ describes a line connecting $\vec{r}_{0}$ and $\vec{r}$. When the object has a constant acoustic speed $c(\vec{r})=c_{0}$, (18) yields $t_{f}\left(\vec{r}, \vec{r}_{0}\right)=\mid \vec{r}_{0}-$ $\vec{r} \mid / c_{0}$, and (17) reduces to (1).

If the acoustic speed distribution $c(\vec{r})$ is known, the TOF function $t_{f}\left(\vec{r}, \vec{r}_{0}\right)$ can be determined by use of (18) and one can attempt to invert (17) to determine $A(\vec{r})$ exactly. However, in current applications of TAT $c(\vec{r})$ is not available and images are reconstructed by use of algorithms that assume a constant acoustic speed. As described next, the image artifacts that arise due to ignoring acoustic heterogeneities can be mitigated, in many cases, by use of a half-time reconstruction method.

We will consider that the image $A(\vec{r})$ is reconstructed by the method described in Section II. In analogy with (2), we form the data function

$$
\hat{g}\left(\vec{r}_{0}, \bar{t}\right)=\frac{4 \pi}{\eta} t \int_{0}^{t} d t_{0} \hat{p}\left(\vec{r}_{0}, t_{0}\right) .
$$

(Because the phenomenon described below do not rely on sampling effects, we will consider here the continuous versions of the data functions.) The data function $\hat{g}\left(\vec{r}_{0}, \bar{t}\right)$ is then processed by a (full-time) reflectivity tomography reconstruction algorithm for reconstruction of an image $\hat{A}(\vec{r})$. However, because 


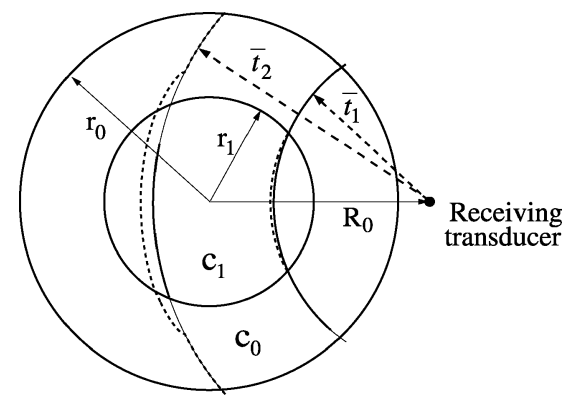

Fig. 3. A schematic of the heterogeneous acoustic velocity distribution $c(\vec{r})$. The value $c_{0}$ denotes the (known) background velocity while $c_{1}$ denotes the velocity of the heterogeneous region. Superimposed on the schematic are contours that represent locations having constant optical path lengths to the receiver position for the cases $c_{1}=c_{0}$ (solid lines) and $c_{1}=1.0759 c_{0}$ (dashed lines). Please refer to Section IV-B for a complete description.

the data $\hat{g}\left(\vec{r}_{0}, \bar{t}\right)$ is inconsistent with respect to the imaging model in $(2), \hat{A}(\vec{r}) \neq A(\vec{r})$ and $\hat{A}(\vec{r})$ can contain significant artifacts and distortions. It is easy to understand the origin of these data inconsistencies. On substitution from (17) into (19) one finds that

$$
\hat{g}\left(\vec{r}_{0}, \bar{t}\right)=\int d^{3} \vec{r} A(\vec{r}) \delta\left[\bar{t}-c_{0} t_{f}\left(\vec{r}, \vec{r}_{0}\right)\right] \frac{c_{0} t_{f}\left(\vec{r}, \vec{r}_{0}\right)}{\left|\vec{r}_{0}-\vec{r}\right|} .
$$

For a given value of $\bar{t}, \hat{g}\left(\vec{r}_{0}, \bar{t}\right)$ represents a weighted integral of $A(\vec{r})$ over the surface $\bar{t}=c_{0} t_{f}\left(\vec{r}, \vec{r}_{0}\right)$ that is not spherical generally. Accordingly, $\hat{g}\left(\vec{r}_{0}, \bar{t}\right)$ and $g\left(\vec{r}_{0}, \bar{t}\right)$ contain different information regarding $A(\vec{r})$. When $c(\vec{r})=c_{0}$ and therefore $t_{f}\left(\vec{r}, \vec{r}_{0}\right)=\left|\vec{r}_{0}-\vec{r}\right| / c_{0},(20)$ reduces to (2).

\section{B. Half-Time Reconstruction Approach}

Let $\hat{g}^{(1)}\left(\vec{r}_{0}, \bar{t}\right)$ and $\hat{g}^{(2)}\left(\vec{r}_{0}, \bar{t}\right)$ denote half-time data functions that are defined from $\hat{g}\left(\vec{r}_{0}, \bar{t}\right)$ in analogy with (3) and (4). For many objects of practical interest, the use of a half-time reconstruction method that employs $\hat{g}^{(1)}\left(\vec{r}_{0}, \bar{t}\right)$ can reduce the severity of the distortions and artifacts in $\hat{A}(\vec{r})$. To understand this heuristically, we can examine the 2-D problem and consider the acoustic velocity distribution shown in Fig. 3. This distribution is comprised of two uniform concentric regions that have acoustic velocities $c_{0}$ and $c_{1}$, with $c_{0} \neq c_{1}$, and radii $r_{0}$ and $r_{1}$, respectively. This type of heterogeneous velocity distribution could represent an acoustic model of the female breast [4], for example. We will assume that the homogeneous matching medium (that the object is embedded in) also has an acoustic velocity $c_{0}$.

As discussed above, the acoustic heterogeneity, which in Fig. 3 is represented by the inner disk with $c(\vec{r})=c_{1}$, will cause the data functions $g\left(\vec{r}_{0}, \bar{t}\right)$ and $\hat{g}\left(\vec{r}_{0}, \bar{t}\right)$ to differ. The key observation is that the magnitude of this difference will depend on the value of $\bar{t}$. More specifically, the magnitude of the difference will be smaller, in general, for small values of $\bar{t}$ than for large values of $\bar{t}$. This is because $\left|t_{f}\left(\vec{r}, \vec{r}_{0}\right)-\right| \vec{r}_{0}-\vec{r}\left|/ c_{0}\right|$ will become generally larger as the path-length through the acoustic heterogeneity that the pressure wavefield must propagate increases. For example, as seen in Fig. 3, for $\bar{t}<R_{0}-r_{1}$ the acoustic heterogeneity is not encountered and therefore $g\left(\vec{r}_{0}, \bar{t}\right)=\hat{g}\left(\vec{r}_{0}, \bar{t}\right)$. For $\bar{t} \geq R_{0}-r_{1}$, the acoustic heterogeneity has an impact on the TOF and therefore the curve $\bar{t}=c_{0} t_{f}\left(\vec{r}, \vec{r}_{0}\right)$ becomes distorted from the circular curve $\bar{t}=\left|\vec{r}_{0}-\vec{r}\right| / c_{0}$, and the degree of this distortion increases with increasing $\bar{t}$. This effect is demonstrated in Fig. 3 for two different values of $\bar{t}$. The solid and dashed lines represent the curves $\bar{t}=\left|\vec{r}_{0}-\vec{r}\right| / c_{0}$ and $\bar{t}=c_{0} t_{f}\left(\vec{r}, \vec{r}_{0}\right)$ that were calculated analytically assuming the values $c_{1} / c_{0}=1.07, r_{0}=0.92$, $r_{1}=0.54$, and $R_{0}=1.2$ (arbitrary units), respectively.

These observations indicate that, for a large class of objects, when acoustic heterogeneities are present the data $\hat{g}\left(\vec{r}_{0}, \bar{t}\right)$ will become less spherical Radon transform-like (or less circular Radon transform-like in the 2-D case) as $\bar{t}$ increases. Therefore, the magnitude of the inconsistent component of $\hat{g}\left(\vec{r}_{0}, \bar{t}\right)$ will become larger with increasing $\bar{t}$. This suggests that a half-time reconstruction method that employs $\hat{g}^{(1)}\left(\vec{r}_{0}, \bar{t}\right)$ can produce images with reduced artifact and distortion levels than contained in images reconstructed from $\hat{g}^{(2)}\left(\vec{r}_{0}, \bar{t}\right)$ or $\hat{g}\left(\vec{r}_{0}, \bar{t}\right)$. This effect is demonstrated clearly by the numerical results in Section VI.

\section{NUMERICAL STUDIES}

We performed numerical studies using simulation and experimental TAT measurement data to investigate the half-time-based reconstruction approaches for TAT discussed in Sections III and IV. Although both the simulation and experimental data corresponded to 2-D TAT measurement geometries, the conclusions derived from these studies are applicable immediately to the 3-D case.

\section{A. Half-Time-Based Methods for Controlling Image Variance}

We investigated quantitatively the statistical properties of the half-time-based reconstruction methods discussed in Section III. In these studies, it was assumed that the acoustic properties of the object were homogeneous.

Simulated Noiseless TAT Measurement Data: The 2-D numerical phantom shown in Fig. 4, left panel, was chosen to represent $A(\vec{r})$. From knowledge of $A(\vec{r})$, which had a radius of $R_{f}=1$ (arbitrary units), the (full-time) noiseless data function $g\left[\phi_{k}, \bar{t}_{l}\right]$ was calculated numerically by use of (5) assuming the 2-D measurement geometry shown in Fig. 1 with $R_{0}=1.2$. Here, the angle $\phi_{k}$ replaces $\vec{r}_{0}^{k}$ as the indicator of the ultrasound receiver position on the circular scanning aperture. The data function $g\left[\phi_{k}, \bar{t}_{l}\right]$ was determined at $N_{\phi}=180$ equally spaced values of $\phi_{k}$ on the scanning aperture over the interval $[0,2 \pi)$ and at $N_{\bar{t}}=129$ equally spaced values of $\bar{t}_{l}$ over the interval $\left[R_{0}-R_{f}, R_{0}+R_{f}\right]$.

Half-time data functions $g^{(1)}\left[\phi_{k}, \bar{t}_{l}\right]$ and $g^{(2)}\left[\phi_{k}, \bar{t}_{l}\right]$ were defined $\forall \phi_{k}$ as

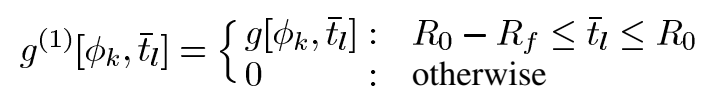

and

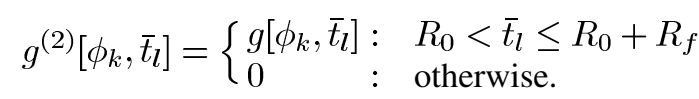




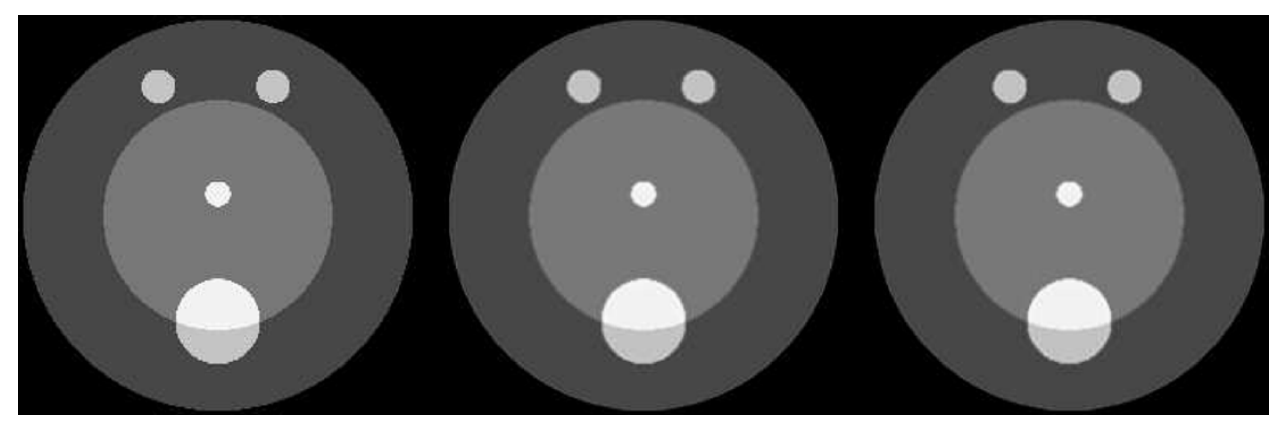

Fig. 4. The numerical phantom for $A(\vec{r})$ employed in our simulation studies is shown in the left panel. The center and right panels display images reconstructed from the noiseless data functions $g\left[\phi_{k} ; \bar{t}_{l}\right]$ and $g^{(1)}\left[\phi_{k} ; \bar{t}_{l}\right]$, respectively.

Noise Models: To generate noisy data functions, $g\left[\phi_{k} ; \bar{t}_{l}\right]$ was treated as a realization of a stochastic process. According to (5) and (6), $\boldsymbol{g}\left[\phi_{k}, \bar{t}_{l}\right]$ can be expressed as

$$
\boldsymbol{g}\left[\phi_{k}, \bar{t}_{l}\right]=g\left[\phi_{k}, \bar{t}_{l}\right]+\frac{4 \pi \Delta t}{\eta c_{0}} \bar{t}_{l} \sum_{m=0}^{l} \boldsymbol{n}\left[\phi_{k}, t_{m}\right] .
$$

For convenience, in our simulations the data were normalized in such a way that $4 \pi \Delta t / \eta c_{0} \equiv 1$. The noise processes $\boldsymbol{n}\left(\vec{r}_{0}^{k}, t_{m}\right)$ were treated as realizations of zero-mean uncorrelated Gaussian stochastic processes with variances chosen according to the rules

$$
\sigma_{n}^{2}=(0.03)^{2}\left(t_{m}\right)^{a}
$$

where $a=0,2$, or 3 . This family of noise models can describe electronic noise contributed by the detector system and other stochastic effects that contaminate the data function.

Reconstruction Algorithm: Analytic inversion formulae for reconstructing images in TAT from half-time data functions have not been identified. However, because (5) is a positive integral equation, the expectation maximization (EM) algorithm [11], [17] could be employed for reconstruction of $A(\vec{r})$. A desirable characteristic of the EM algorithm is that it ensures the nonnegativity of the reconstructed image $A(\vec{r})$, which is required by the physics of the problem. The EM algorithm was employed for reconstruction of the images $\boldsymbol{A}^{(1)}(\vec{r}), \boldsymbol{A}^{(2)}(\vec{r})$, and $\boldsymbol{A}(\vec{r})$ from the data functions $\boldsymbol{g}^{(1)}\left[\phi_{k}, \bar{t}_{l}\right], \boldsymbol{g}^{(2)}\left[\phi_{k}, \bar{t}_{l}\right]$, and $g\left[\phi_{k}, \bar{t}_{l}\right]$, respectively. The matrix size of the reconstructed images was $128 \times 128$ pixels. Details describing the application of the EM algorithm in reflectivity tomography can be found in [11].

Empirical Determination of Image Statistics: If the second-order statistics of the data $\boldsymbol{g}\left[\phi_{k}, \bar{t}_{l}\right]$ are known, one can attempt to calculate [18], [19] the second-order statistics that are required for determination of the optimal combination $\omega_{i, j}^{o p t}$ in (16) for a given family of estimators (i.e., a given $i$ and $j$ ). In this work, we conducted Monte Carlo studies for determination of empirical estimates of these statistical properties. Using the noise models given in (23) and (24), $N=600$ noisy realizations of the data functions $\boldsymbol{g}^{(1)}\left[\phi_{k}, \bar{t}_{l}\right], \boldsymbol{g}^{(2)}\left[\phi_{k}, \bar{t}_{l}\right]$, and $g\left[\phi_{k}, \bar{t}_{l}\right]$ were generated. From these ensembles of data functions, corresponding sets of noisy images $\left\{\boldsymbol{A}^{(1)}(\vec{r})\right\}$, $\left\{\boldsymbol{A}^{(2)}(\vec{r})\right\}$, and $\{\boldsymbol{A}(\vec{r})\}$ were reconstructed by use of the EM algorithm. Because an ad-hoc stopping rule was not employed with the EM algorithm, we saved the reconstructed images at various stages of convergence for analysis. The sets of reconstructed images were used to determine empirical estimates [20] of $\operatorname{Var}\left\{\boldsymbol{A}^{(1)}(\vec{r})\right\}, \operatorname{Var}\left\{\boldsymbol{A}^{(2)}(\vec{r})\right\}$, and $\operatorname{Var}\{\boldsymbol{A}(\vec{r})\}$. Empirical estimates of the covariances $\operatorname{Covar}\left\{\boldsymbol{A}^{(1)}(\vec{r}), \boldsymbol{A}^{(2)}(\vec{r})\right\}$ and $\operatorname{Covar}\left\{\boldsymbol{A}^{(1)}(\vec{r}), \boldsymbol{A}(\vec{r})\right\}$ were determined also. These statistical quantities were used in (16) to determine estimates of $\omega_{1,2}^{o p t}$ and $\omega_{1,3}^{o p t}$.

Subsequent to the determination of the optimal combination coefficients, we conducted simulation studies to investigate the statistical properties of the estimators in (13). From the sets of noisy images $\left\{\boldsymbol{A}^{(1)}(\vec{r})\right\},\left\{\boldsymbol{A}^{(2)}(\vec{r})\right\}$, and $\{\boldsymbol{A}(\vec{r})\}$ described above, sets of noisy images $\left\{\boldsymbol{A}_{1,2}^{\omega}(\vec{r})\right\}$, and $\left\{\boldsymbol{A}_{1,3}^{\omega}(\vec{r})\right\}$ were generated from (13) as

$$
\boldsymbol{A}_{1,2}^{\omega}(\vec{r})=\omega_{1,2}^{o p t}(\vec{r}) \boldsymbol{A}^{(1)}(\vec{r})+\left[1-\omega_{1,2}^{o p t}(\vec{r})\right] \boldsymbol{A}^{(2)}(\vec{r})
$$

and

$$
\boldsymbol{A}_{1,3}^{\omega}(\vec{r})=\omega_{1,3}^{o p t}(\vec{r}) \boldsymbol{A}^{(1)}(\vec{r})+\left[1-\omega_{1,3}^{o p t}(\vec{r})\right] \boldsymbol{A}(\vec{r})
$$

respectively. The sets of reconstructed images $\left\{\boldsymbol{A}_{1,2}^{\omega}(\vec{r})\right\}$ and $\left\{\boldsymbol{A}_{1,3}^{\omega}(\vec{r})\right\}$ were used to determine empirical estimates of $\operatorname{Var}\left\{\boldsymbol{A}_{1,2}^{\omega}(\vec{r})\right\}$, and $\operatorname{Var}\left\{\boldsymbol{A}_{1,3}^{\omega}(\vec{r})\right\}$.

\section{B. Mitigation of Artifacts From Acoustic Heterogeneities}

We performed numerical studies to demonstrate that a halftime reconstruction approach that utilizes $g^{(1)}\left[\phi_{k}, \bar{t}_{l}\right]$ can mitigate artifacts that arise from heterogeneities in the acoustic properties of the object. Simulated 2-D measurement data were generated according to (the 2-D version of) (20). The object $A(\vec{r})$ was the same as utilized in Section V-A (see Fig. 4, left panel) and the heterogeneous acoustic speed distribution $c(\vec{r})$ was the same as described in Section IV-B (see Fig. 3). The data function $\hat{g}\left[\phi_{k}, \bar{t}_{l}\right]$ was determined at $N_{\phi}=360$ equally spaced values of $\phi_{k}$ on the circular scanning aperture over the interval $[0,2 \pi)$ and at $N_{\bar{t}}=257$ equally spaced values of $\bar{t}_{l}$ over the interval $\left[R_{0}-R_{f}, R_{0}+R_{f}\right]$. The computation of $\hat{g}\left[\phi_{k}, \bar{t}_{l}\right]$ involved the following three steps. First, for a given value of $\bar{t}_{l}$, sets of evenly sampled points $\left\{\vec{r}_{i}\right\}$ that reside on the curves $\bar{t}_{l}=c_{0} t_{f}\left(\vec{r}_{i}, \vec{r}_{0}\right)$ were determined analytically. Second, bilinear interpolation was utilized to determine the values of $A\left(\vec{r}_{i}\right)$ at the locations $\left\{\vec{r}_{i}\right\}$. Finally, the values of $A\left(\vec{r}_{i}\right)$ were weighted by the factor $c_{0} t_{f}\left(\vec{r}_{i}, \vec{r}_{0}\right) /\left|\vec{r}_{0}-\vec{r}_{i}\right|$ and the 2-D version of (20) was approximated by use of trapezoidal integration. Variations in the definition of the acoustic speed distribution $c(\vec{r})$ were considered and multiple data sets were generated. 


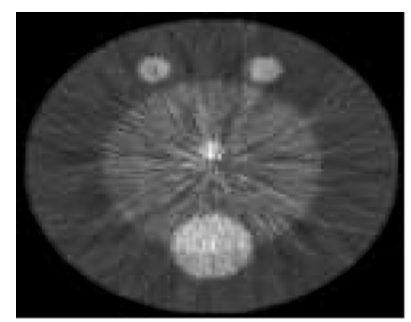

(a)

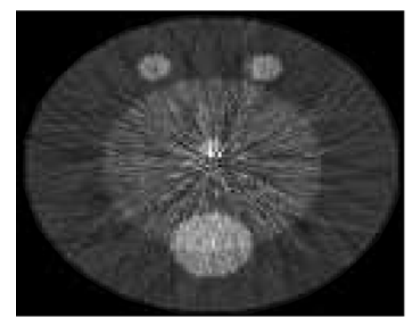

(d)

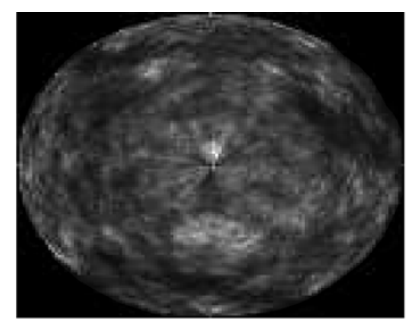

(b)

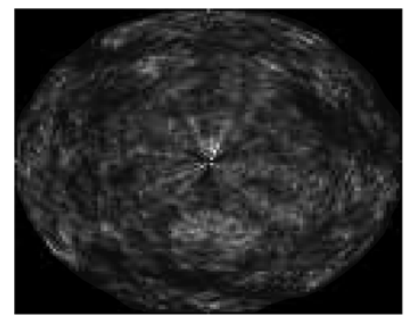

(e)

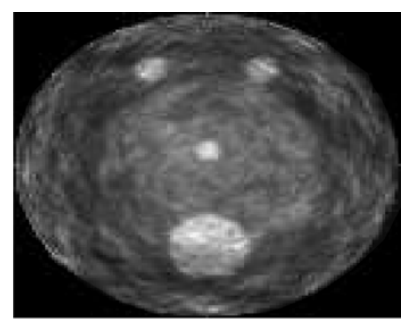

(c)

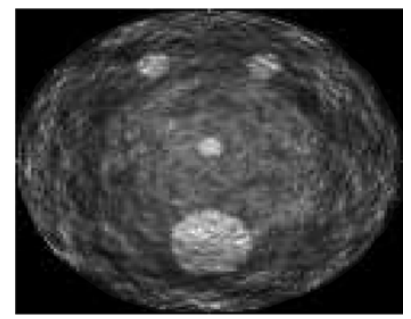

(f)

Fig. 5. Reconstructed images corresponding to the noise model in (24) with $a=3$. Images in (a)-(c) were reconstructed from noisy realizations of the data functions $\boldsymbol{g}^{(1)}\left[\phi_{k}, \bar{t}_{l}\right], \boldsymbol{g}^{(2)}\left[\phi_{k}, \bar{t}_{l}\right]$, and $\boldsymbol{g}\left[\phi_{k}, \bar{t}_{l}\right]$, respectively, by use of the EM algorithm with 40 iterations. Panels (d)-(f) contain the corresponding images reconstructed by use of 80 iterations of the EM algorithm.

Half-time data functions $\hat{g}^{(1)}\left[\phi_{k}, \bar{t}_{l}\right]$ were defined in analogy with (21). The EM algorithm was employed for reconstruction of the images $\hat{A}^{(1)}(\vec{r})$ and $\hat{A}(\vec{r})$ from the data functions $\hat{g}^{(1)}\left[\phi_{k}, \bar{t}_{l}\right]$ and $\hat{g}\left[\phi_{k}, \bar{t}_{l}\right]$, respectively. The matrix size of the reconstructed images was $256 \times 256$ pixels.

\section{Experimental Data}

We also reconstructed images from two sets of experimental TAT measurement data that were acquired previously in the Optical Imaging Laboratory at Texas A\&M University. ${ }^{2}$ The first data set corresponded to a physical phantom that is described in [3]. The data were acquired using a microwave source and a 2-D measurement geometry that had a scanning radius $R_{0}=70 \mathrm{~mm}$ [3]. Measurements were taken at 160 equally spaced positions on the scanning aperture and for each measurement the received pressure signal was sampled at 2000 points at a sampling frequency of $50 \mathrm{MHz}$. The second data set corresponded to a mouse brain that was imaged by use of a TAT animal scanner [21]. The data were acquired using an optical source and a 2-D measurement geometry that had a scanning radius $R_{0}=19.95 \mathrm{~mm}$ [21]. Measurements were taken at 240 equally spaced positions on the scanning aperture and for each measurement the received pressure signal was sampled at 5000 points at a rate of 100 samples/microsecond. Full- and half-time data reflectivity tomography data functions were calculated from the recorded pressure signals by use of (5) and corresponding images were reconstructed via the EM algorithm with 100 iterations.

\section{NUMERICAL RESULTS}

\section{A. Statistical Properties of Half-Time-Based Methods}

Fig. 4 contains images reconstructed from the noiseless data functions $g\left[\phi_{k} ; \bar{t}_{l}\right]$ (center panel) and $g^{(1)}\left[\phi_{k} ; \bar{t}_{l}\right]$ (right panel). Both of these images appear virtually identical to the true

${ }^{2}$ Professor Lihong V. Wang, Optical Imaging Laboratory, Department of Biomedical Engineering, Texas A\&M University, College Station, TX.

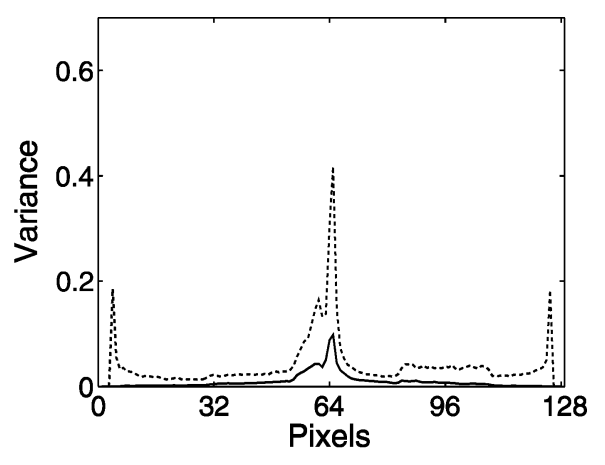

(a)

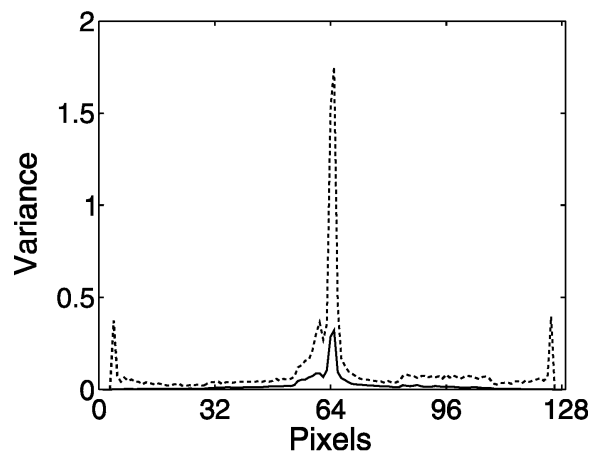

(b)

Fig. 6. Vertical profiles through the centers of the empirical variance maps $\operatorname{Var}\left\{\boldsymbol{A}^{(1)}(\vec{r})\right\}$ (solid line) and $\operatorname{Var}\left\{A^{(2)}(\vec{r})\right\}$ (dashed line) for the noise model in (24) with $a=3$. Panels (a) and (b) correspond to images obtained by use of the EM algorithm with 40 and 80 iterations, respectively.

phantom that is shown in the left panel of the same figure. This is consistent with our assertion that accurate TAT images can be reconstructed from the half-time data function $g^{(1)}\left[\phi_{k} ; \bar{t}_{l}\right]$. These images were reconstructed using 200 iterations of the EM algorithm.

Noise Model: $\sigma_{n}=(0.03)^{2}\left(t_{m}\right)^{3}$ : Figs. (5)-(8) contain results that correspond to the noise model in (24) with $a=3$. 


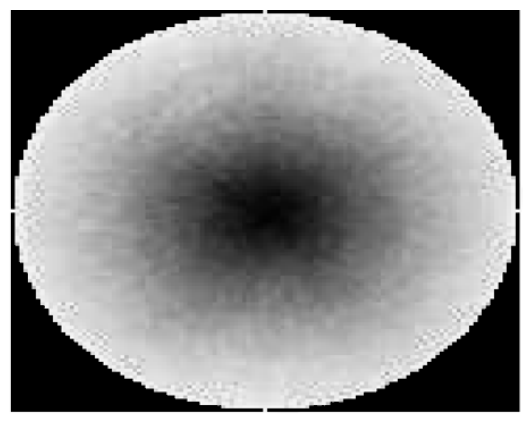

(a)

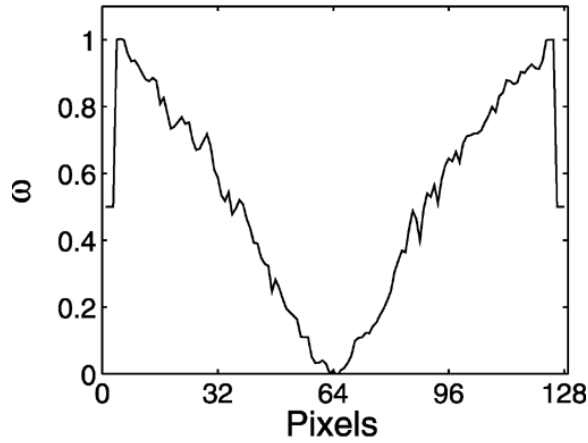

(b)

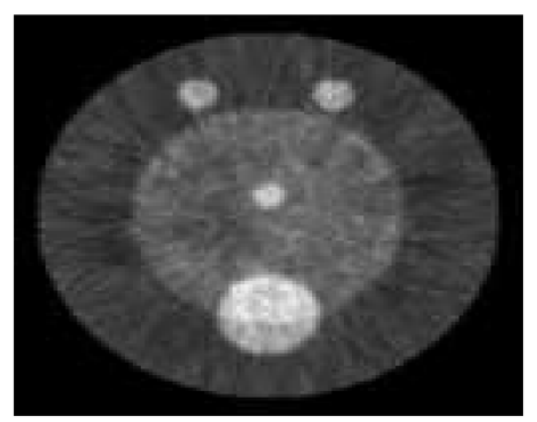

(c)

Fig. 7. (a) $\omega_{1,3}^{o p t}(x, y)$ computed for the noise model in (24) with $a=3$. (b) A vertical profile through the center of $\omega_{1,3}^{o p t}(x, y)$. (c) An image $\boldsymbol{A}_{1,3}^{\omega}(\vec{r})$ formed by use of (26) from noisy realizations of $\boldsymbol{A}^{(1)}(\vec{r})$ and $\boldsymbol{A}(\vec{r})$.

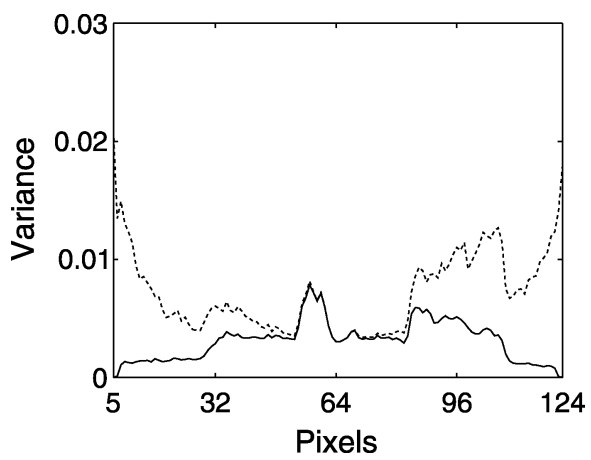

Fig. 8. Vertical profiles through the centers of the empirical variance maps $\operatorname{Var}\left\{\boldsymbol{A}_{1,3}^{\omega}(\vec{r})\right\}$ (solid line) and $\operatorname{Var}\{\boldsymbol{A}(\vec{r})\}$ (dashed line) for the noise model in (24) with $a=3$.

The images in Fig. 5(a)-(c) were reconstructed from noisy realizations of the data functions $\boldsymbol{g}^{(1)}\left[\phi_{k}, \bar{t}_{l}\right], \boldsymbol{g}^{(2)}\left[\phi_{k}, \bar{t}_{l}\right]$, and $g\left[\phi_{k}, \bar{t}_{l}\right]$, respectively, by use of the EM algorithm with 40 iterations. Fig. 5(d)-(f) shows the corresponding images reconstructed by use of 80 iterations of the EM algorithm. It appears that the images reconstructed from the data function $\boldsymbol{g}^{(1)}\left[\phi_{k}, \bar{t}_{l}\right]$ [Fig. 5(a) and (d)] contain significantly reduced noise levels as compared to the corresponding images reconstructed from the data function $\boldsymbol{g}^{(2)}\left[\phi_{k}, \bar{t}_{l}\right]$ [(Fig. 5(b) and (e)]. These observations are confirmed quantitatively by the variance plots in Fig. 6. Fig. 6(a) contains vertical profiles through the centers of the empirical variance maps $\operatorname{Var}\left\{\boldsymbol{A}^{(1)}(\vec{r})\right\}$ (solid line) and $\operatorname{Var}\left\{\boldsymbol{A}^{(2)}(\vec{r})\right\}$ (dashed line) computed from the ensemble of images obtained by use of the EM algorithm with 40 iterations. The results corresponding to images reconstructed by use of the EM algorithm with 80 iterations are shown in Fig. 6(b). In both cases it is clear that $\operatorname{Var}\left\{\boldsymbol{A}^{(1)}(\vec{r})\right\}<\operatorname{Var}\left\{\boldsymbol{A}^{(2)}(\vec{r})\right\}$, which is consistent with the discussions in Sections III-A and III-B.

It also appears that the images reconstructed from the data function $\boldsymbol{g}^{(1)}\left[\phi_{k}, \bar{t}_{l}\right]$ [Fig. 5(a) and (d)] contain significantly reduced noise levels in regions away from the image center as compared to the corresponding images reconstructed from the full-time data function $\boldsymbol{g}\left[\phi_{k}, \bar{t}_{l}\right]$ [Fig. 5(c) and (f)]. This is confirmed by comparison of the variance plots in Figs. 6 and 8. The dashed curve in Fig. 8 represents a vertical profile through the center of the empirical variance map $\operatorname{Var}\{\boldsymbol{A}(\vec{r})\}$ computed from the ensemble of images obtained by use of the EM algorithm with 40 iterations. (The solid line in Fig. 8 will be discussed below.) Figs. 6 and 8 indicate that near the center of image space $\operatorname{Var}\{\boldsymbol{A}(\vec{r})\}<\operatorname{Var}\left\{\boldsymbol{A}^{(1)}(\vec{r})\right\}$, but $\operatorname{Var}\left\{\boldsymbol{A}^{(1)}(\vec{r})\right\}<\operatorname{Var}\{\boldsymbol{A}(\vec{r})\}$ in the outer regions of image space. These results hold true also for images obtained by use of the EM algorithm with 80 iterations.

The observations above suggest that it is highly desirable to form the image $\boldsymbol{A}_{1,3}^{\omega}(\vec{r})$ defined in (26). This is because the behavior of $\operatorname{Var}\{\boldsymbol{A}(\vec{r})\}$ is complementary to that of $\operatorname{Var}\left\{\boldsymbol{A}^{(1)}(\vec{r})\right\}$ and this can be exploited by use of the combination coefficient $\omega_{1,3}^{o p t}$. For $\boldsymbol{A}^{(1)}(\vec{r})$ and $\boldsymbol{A}(\vec{r})$ computed by use of the EM algorithm with 40 iterations, $\omega_{1,3}^{o p t}(x, y)$ and a vertical profile through the center of $\omega_{1,3}^{o p t}(x, y)$ are shown in Fig. 7(a) and (b), respectively. The combination coefficient $\omega_{1,3}^{o p t}(x, y)$ decreases from a value near 1 at the edge of image space down to a value near 0 at the center of image space. This reflects that $\boldsymbol{A}^{(1)}(\vec{r})$ is weighted to contribute more to $\boldsymbol{A}_{1,3}^{\omega}(\vec{r})$ away from the center of image space where $\operatorname{Var}\left\{\boldsymbol{A}^{(1)}(\vec{r})\right\}<\operatorname{Var}\{\boldsymbol{A}(\vec{r})\}$. Near the center of image space, $\operatorname{Var}\left\{\boldsymbol{A}^{(1)}(\vec{r})\right\}>\operatorname{Var}\{\boldsymbol{A}(\vec{r})\}$ and therefore $\boldsymbol{A}(\vec{r})$ is weighted to contribute more to $\boldsymbol{A}_{1,3}^{\omega}(\vec{r})$. Fig. 7(c) contains an image $A_{1,3}^{\omega}(\vec{r})$ formed by use of (26) from noisy realizations of $\boldsymbol{A}^{(1)}(\vec{r})$ and $\boldsymbol{A}(\vec{r})$. The solid curve in Fig. 8 represents a vertical profile through the center of the empirical variance map $\operatorname{Var}\left\{\boldsymbol{A}_{1,3}^{\omega}(\vec{r})\right\}$. This curve is lower in most places (and nowhere higher) than the dashed curve that represents a profile through $\operatorname{Var}\{\boldsymbol{A}(\vec{r})\}$. Therefore, the magnitude of the noise in images $\boldsymbol{A}_{1,3}^{\omega}(\vec{r})$ will be smaller, on average, than in images $\boldsymbol{A}(\vec{r})$. Moreover, as expected, $\operatorname{Var}\left\{\boldsymbol{A}_{1,3}^{\omega}(\vec{r})\right\} \leq \operatorname{Var}\left\{\mathrm{A}^{(1)}(\vec{r})\right\}$ everywhere. Qualitatively similar results were obtained for images reconstructed by the EM algorithm with 80 iterations.

Because, as shown in Fig. 6, both $\operatorname{Var}\left\{\boldsymbol{A}^{(1)}(\vec{r})\right\}$ and $\operatorname{Var}\left\{\boldsymbol{A}^{(2)}(\vec{r})\right\}$ are large near the center of image space, the image $\boldsymbol{A}_{1,2}^{\omega}(\vec{r})$ can be expected to have enhanced noise levels 


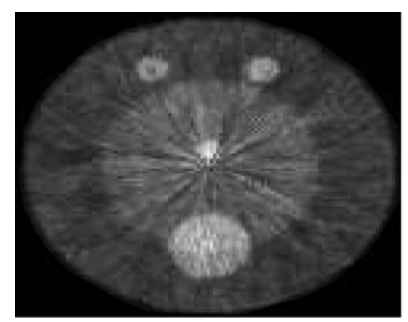

(a)

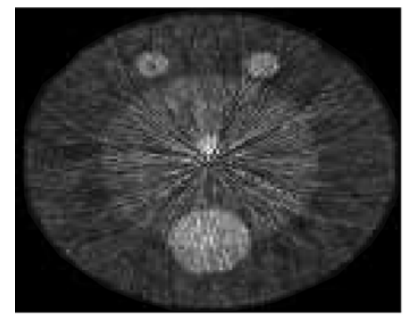

(d)

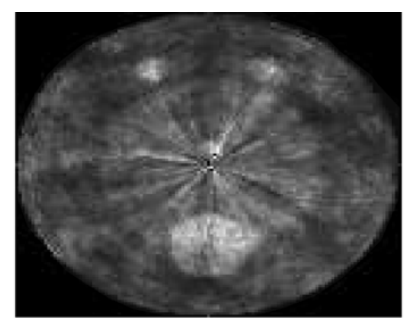

(b)

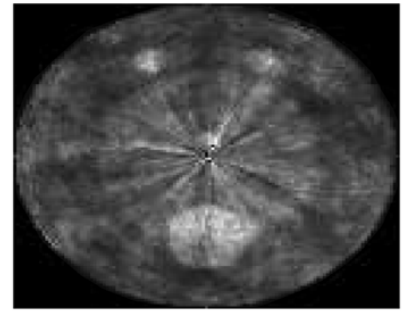

(e)

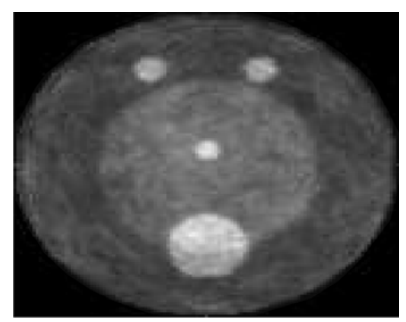

(c)

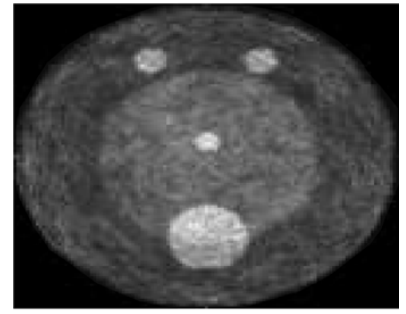

(f)

Fig. 9. Reconstructed images corresponding to the noise model in (24) with $a=0$. Images in figures (a)-(c) were reconstructed from noisy realizations of the data functions $\boldsymbol{g}^{(1)}\left[\phi_{k}, \bar{t}_{l}\right], \boldsymbol{g}^{(2)}\left[\phi_{k}, \bar{t}_{l}\right]$, and $\boldsymbol{g}\left[\phi_{k}, \bar{t}_{l}\right]$, respectively, by use of the EM algorithm with 40 iterations. Figures (d)-(f) contain the corresponding images reconstructed by use of 80 iterations of the EM algorithm.

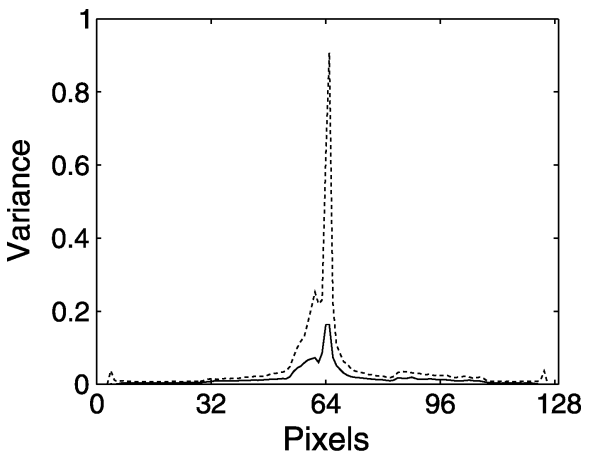

(a)

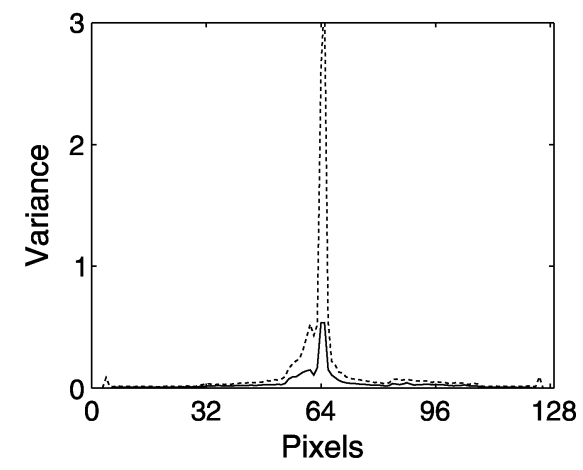

(b)

Fig. 10. Vertical profiles through the centers of the empirical variance maps $\operatorname{Var}\left\{A^{(1)}(\vec{r})\right\}$ (solid line) and $\operatorname{Var}\left\{A^{(2)}(\vec{r})\right\}$ (dashed line) for the noise model in (24) with $a=0$. Panels (a) and (b) correspond to images obtained by use of the EM algorithm with 40 and 80 iterations, respectively.

in that region. Although not shown, this prediction was verified in our numerical simulations.

Noise Model: $\sigma_{n}=(0.03)^{2}$ : Figs. (9)-(12) contain results that correspond to the noise model in (24) with $a=0$. The images in Fig. 9(a)-(c) were reconstructed from noisy realizations of the data functions $\boldsymbol{g}^{(1)}\left[\phi_{k}, \bar{t}_{l}\right], \boldsymbol{g}^{(2)}\left[\phi_{k}, \bar{t}_{l}\right]$, and $\boldsymbol{g}\left[\phi_{k}, \bar{t}_{l}\right]$, respectively, by use of the EM algorithm with 40 iterations. Fig. 9(d)-(f) are the corresponding images reconstructed by use of 80 iterations of the EM algorithm. As expected, The images reconstructed from the data function $g^{(1)}\left[\phi_{k}, \bar{t}_{l}\right]$ [Fig. 9(a) and (d)] appear to contain significantly reduced noise levels as compared to the corresponding images reconstructed from the data function $\boldsymbol{g}^{(2)}\left[\phi_{k}, \bar{t}_{l}\right]$ [Fig. 9(b) and (e)]. These observations are confirmed quantitatively by the variance plots in Fig. 10.

The images reconstructed from the data function $\boldsymbol{g}^{(1)}\left[\phi_{k}, \bar{t}_{l}\right]$ [Fig. 9(a) and (d)] appear to contain slightly reduced noise levels in regions away from the image center as compared to the corresponding images reconstructed from the full-time data function $g\left[\phi_{k}, \bar{t}_{l}\right]$ [Fig. 9(c) and (f)], but the effect is not as pronounced as was observed for the noise model with $a=3$ discussed above. As before, we can form the estimate $\boldsymbol{A}_{1,3}^{\omega}(\vec{r})$. For $\boldsymbol{A}^{(1)}(\vec{r})$ and $A(\vec{r})$ computed by use of the EM algorithm with 40 iterations, $\omega_{1,3}^{o p t}(x, y)$ and a vertical profile through the center of $\omega_{1,3}^{o p t}(x, y)$ are shown in Fig. 11(a) and (b), respectively. Fig. 11(c) contains an image $A_{1,3}^{\omega}(\vec{r})$ formed by use of (26) from noisy realizations of $\boldsymbol{A}^{(1)}(\vec{r})$ and $\boldsymbol{A}(\vec{r})$. The solid curve in Fig. 12 represents a vertical profile through the center of the empirical variance map $\operatorname{Var}\left\{\boldsymbol{A}_{1,3}^{\omega}(\vec{r})\right\}$. This curve is lower in most places than the dashed curve that represents a profile through $\operatorname{Var}\{A(\vec{r})\}$. The fact that the difference between the variance curves is not large in most places indicates that the EM algorithm, when applied to the (full-scan) data function $\boldsymbol{g}\left[\phi_{k}, \bar{t}_{l}\right]$ with the $a=0$ noise model, is implicitly utilizing a combination coefficient that is "close" to $\omega_{1,3}^{o p t}$. Qualitatively similar results were obtained for images reconstructed by the EM algorithm with 80 iterations.

Noise Model: $\sigma_{n}=(0.03)^{2}\left(t_{m}\right)^{2}$ : The results that correspond to the noise model in (24) with $a=2$ can be understood as intermediate cases of the results for the $a=3$ and $a=0$ noise models. As before, the images reconstructed from the data function $\boldsymbol{g}^{(1)}\left[\phi_{k}, \bar{t}_{l}\right]$ appear to contain significantly reduced noise levels as compared to the corresponding images reconstructed from the data function $\boldsymbol{g}^{(2)}\left[\phi_{k}, \bar{t}_{l}\right]$. The differences 


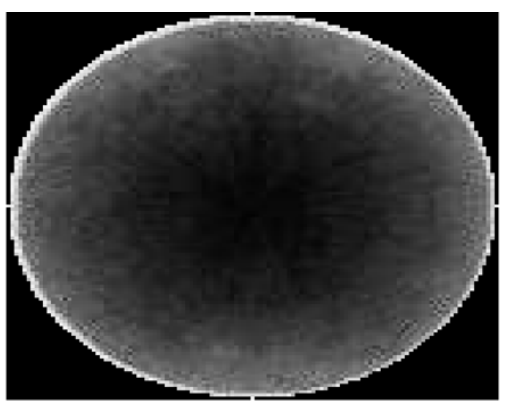

(a)

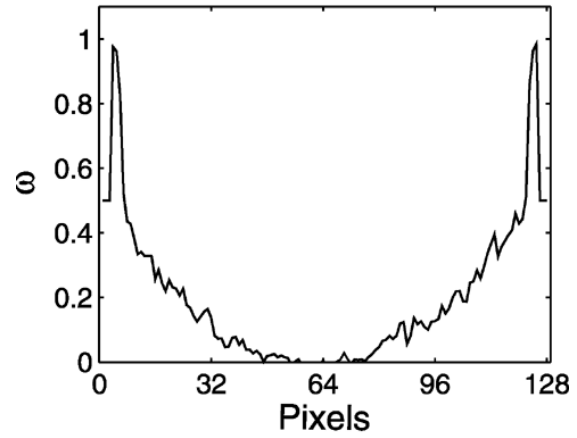

(b)

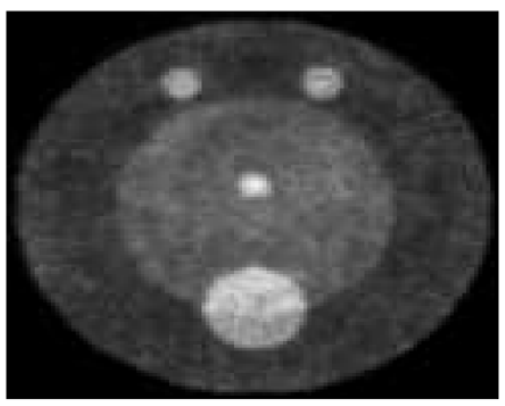

(c)

Fig. 11. (a) $\omega_{1,3}^{\text {opt }}(x, y)$ computed for the noise model in (24) with $a=0$. (b) A vertical profile through the center of $\omega_{1,3}^{o p t}(x, y)$. (c) An image $A_{1,3}^{\omega}(\vec{r})$ formed by use of (26) from noisy realizations of $\boldsymbol{A}^{(1)}(\vec{r})$ and $\boldsymbol{A}(\vec{r})$.

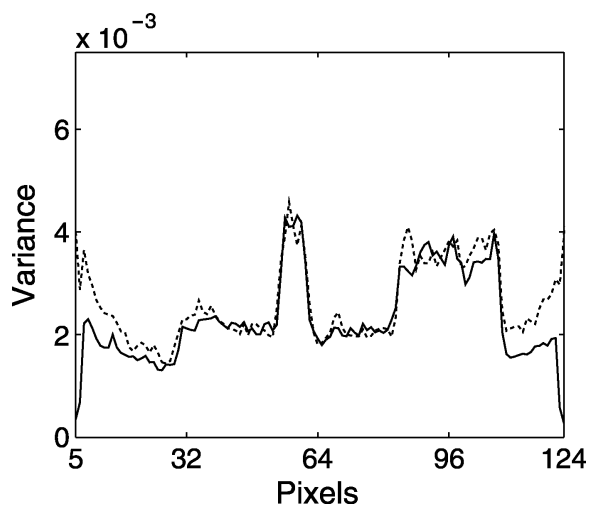

Fig. 12. Vertical profiles through the centers of the empirical variance maps $\operatorname{Var}\left\{\boldsymbol{A}_{1,3}^{\omega}(\vec{r})\right\}$ (solid line) and $\operatorname{Var}\{\boldsymbol{A}(\vec{r})\}$ (dashed line) for the noise model in (24) with $a=0$.

between $\operatorname{Var}\left\{\boldsymbol{A}_{1,3}^{\omega}(\vec{r})\right\}$ and $\operatorname{Var}\{\boldsymbol{A}(\vec{r})\}$ are larger than obtained for the $a=0$ noise model case but smaller than obtained for the $a=3$ noise model case. This indicates that the ability to control the variance properties of $\boldsymbol{A}_{1,3}^{\omega}(\vec{r})$ via (26) [or equivalently

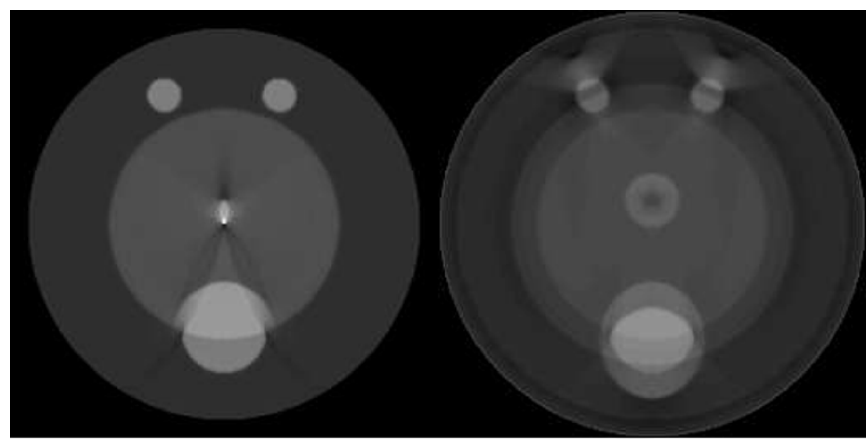

(a)

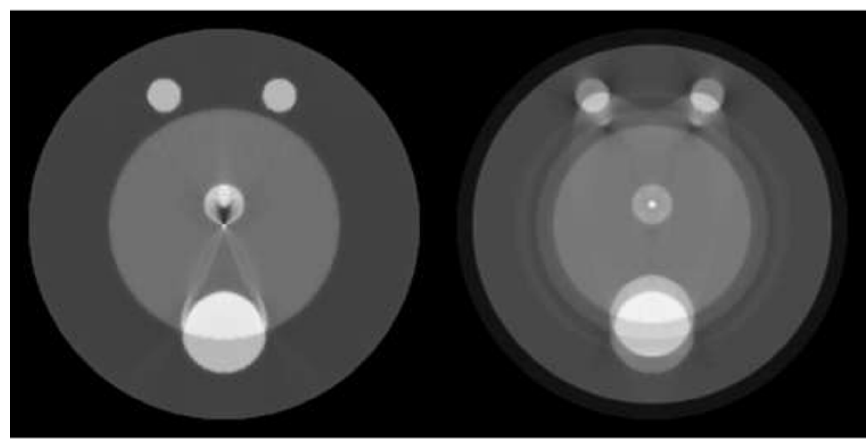

(b)

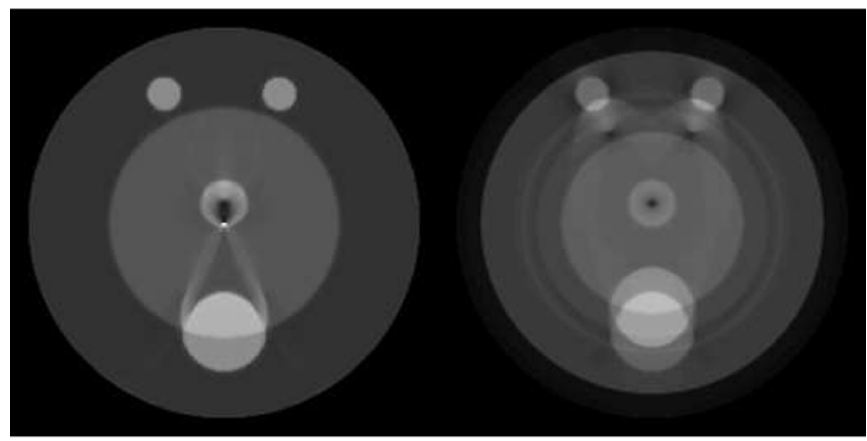

(c)

Fig. 13. Images reconstructed from simulated TAT measurement data containing the effects of acoustic heterogeneities. The images in subfigures (a), (b), and (c) were reconstructed from data corresponding to acoustic velocity map discussed in Section IV-B with $c_{1} / c_{0}=0.9,1.07$, and 1.12 , respectively. The images in the left and right panels of each subfigure were reconstructed from half- and full-time data functions, respectively.

(13)] is particularly important for noise models corresponding to large values $a$.

\section{B. Mitigation of Artifacts Due to Acoustic Heterogeneities}

Fig. 13 contains images reconstructed from simulated TAT measurement data containing the effects of acoustic heterogeneities. The images in subfigures (a), (b), and (c) were reconstructed from data corresponding to acoustic velocity map discussed in Section IV-B with $c_{1} / c_{0}=0.9,1.07$, and 1.12, respectively. In each case, $r_{1}=0.54$ and $r_{0}=0.92$ (arbitrary units). The images in the left and right panels of each subfigure were reconstructed from $\hat{g}^{(1)}\left[\phi_{k}, \bar{t}_{l}\right]$ and $\hat{g}\left[\phi_{k}, \bar{t}_{l}\right]$, respectively. In all cases, the conspicuity of the artifacts and distortions in the images reconstructed from the half-time data $\hat{g}^{(1)}\left[\phi_{k}, \bar{t}_{l}\right]$ is much less than in the images reconstructed from the full-time data $\hat{g}\left[\phi_{k}, \bar{t}_{l}\right]$. This corroborates our assertion in Section IV-B 


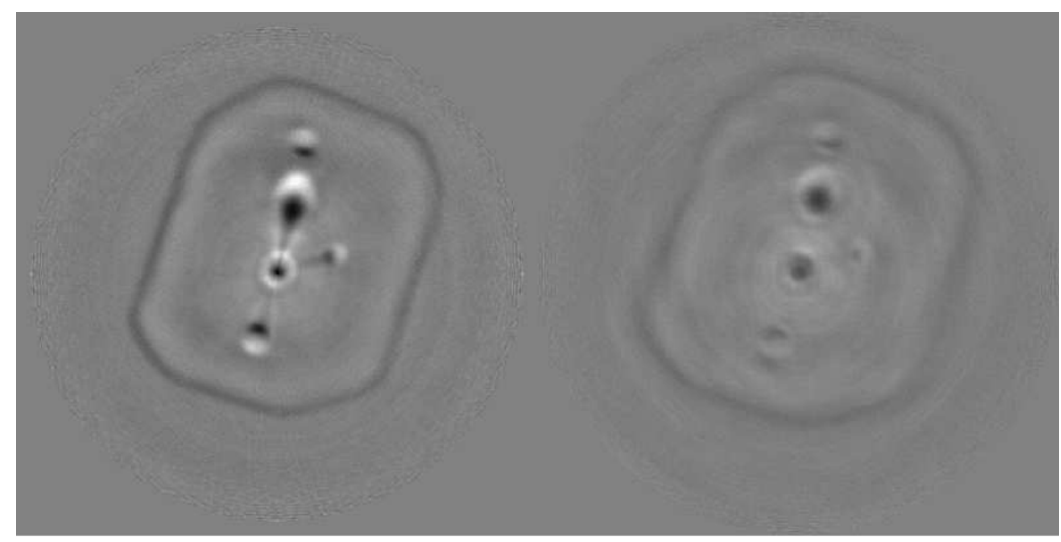

(a)

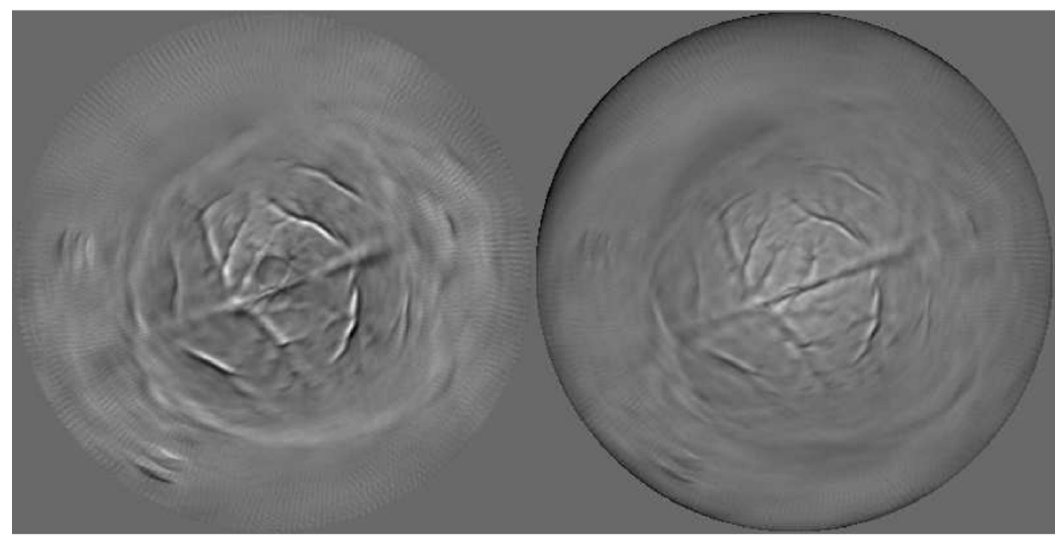

(b)

Fig. 14. Images reconstructed from the experimental TAT data sets that correspond to the (a) physical phantom and (b) mouse brain. The images in the left and right panels were reconstructed from half- and full-time data functions, respectively.

that half-time reconstruction methods can produce images with reduced artifact and distortion levels than contained in images reconstructed from full-time data.

\section{Experimental Results}

Fig. 14 contains the images that were reconstructed from the experimental TAT data sets that correspond to the physical phantom [Fig. 14(a)] and mouse brain [Fig. 14(b)]. The images in the left and right panels were reconstructed from half- and full-time data functions, respectively. Although these particular objects do not possess highly heterogeneous acoustic properties, variations in their acoustic properties produce acoustic reverberations that degrade the fidelity of the measurement data. In both cases, the contrast and resolution of the images reconstructed from half-time data appears to be superior to that of the images reconstructed from the full-time data.

\section{SUMMARY}

Thermoacoustic tomography is an important emerging imaging technique with potential for a wide range of biomedical imaging applications. Although prototype TAT scanners have been built and investigated recently, there remains a great need for the development of accurate and robust tomographic reconstruction algorithms. In this paper, we proposed and investigated reconstruction methods for TAT that were based on the half-time reflectivity tomography paradigm. These reconstruction methods permit for accurate image reconstruction from knowledge of only half of the detected pressure signal at each location on the receiving aperture. This is possible because a complete set of TAT measurement data contains twice as much information as is required theoretically for stable image reconstruction. In essence, the half-time reconstruction problem of reflectivity tomography is analogous to the half-detector problem of X-ray tomography with a parallel-beam tomography. To our knowledge, this is the first work to exploit explicitly such data redundancies in TAT.

We identified and investigated two potential advantages of half-time-based reconstruction methods for TAT. First, we demonstrated that half-time reconstruction methods facilitate the explicit control of statistically complementary information in the TAT measurement data. From a full-time (i.e., complete) set of TAT measurement data, three images can be reconstructed: one from each of the (two) half-time data sets and one from the full-time data set. When the measurement data are noisy, we demonstrated that each of these images will be distinct in general and possess different statistical properties. We proposed and investigated classes of estimation methods that can exploit statistically complementary information contained in these images to form a final image that has an optimally reduced variance. 
Second, we revealed that half-time reconstruction methods can mitigate image artifacts due to heterogeneous acoustic properties of an object. Reconstruction algorithms that are in current use assume that the object is acoustically homogeneous. However, in many applications of TAT, including imaging of the female breast [4], the object of interest may not possess a constant acoustic speed. In these cases, the TAT measurement data will be inconsistent with respect to the imaging model, which can result in image artifacts and distortions. We demonstrated heuristically as well as numerically that a half-time reconstruction method that utilizes the first-half data function $\hat{g}^{(1)}\left(\vec{r}_{0}, \bar{t}\right)$ can reconstruct images with significantly reduced artifact and distortion levels as compared to images reconstructed by use of the second-half or full-time data functions. This results because the temporal components of the pressure wavefields that contribute to the data function $\hat{g}^{(1)}\left(\vec{r}_{0}, \bar{t}\right)$ are often less perturbed by the acoustic heterogeneity than are the components that contribute to the second-half data function $\hat{g}^{(2)}\left(\vec{r}_{0}, \bar{t}\right)$. This is because they are required to propagate through the acoustic heterogeneity over shorter optical path-lengths in general.

Although the EM algorithm was employed for image reconstruction, the perspectives and insights promulgated in this paper are independent of the reconstruction algorithm employed. The investigation of alternative algorithms for reconstruction of TAT images from half-time data functions remains a topic for future studies. Other topics for future studies include the determination of appropriate data noise models for specific experimental TAT scanners and the assessment of how noise propagation characteristics of TAT reconstruction algorithms affect various detections tasks.

\section{REFERENCES}

[1] R. Kruger, P. Liu, R. Fang, and C. Appledorn, "Photoacoustic ultrasound (PAUS) reconstruction tomography," Med. Phys., vol. 22, pp. 1605-1609, 1995.

[2] R. Kruger, D. Reinecke, and G. Kruger, "Thermoacoustic computed tomography-technical considerations," Med. Phys., vol. 26, pp. 1832-1837, 1999.

[3] M. Xu and L. V. Wang, "Time-domain reconstruction for thermoacoustic tomography in a spherical geometry," IIEEE Trans. Med. Imag., vol. 21, no. 7, pp. 814-822, Jul. 2002.
[4] Y. Xu and L. V. Wang, "Effects of acoustic heterogeneity in breast thermoacoustic tomography," IEEE Trans. Ultrason., Ferroelect. Freq. Contr., vol. 50, no. 9, pp. 1134-1146, Sep. 2003.

[5] W. Joines, R. Jirtle, M. Rafal, and D. Schaeffer, "Microwave power absorption differences between normal and malignant tissue," Radiat. Oncol., Biol., Phys., vol. 6, pp. 681-687, 1980.

[6] W. Cheong, S. Prahl, and A. Welch, "A review of the optical properties of biological tissues," IEEE J. Quantum Elect., vol. 26, no. 12, pp. 2166-2185, Dec. 1990.

[7] M. Xu, Y. Xu, and L. V. Wang, "Time-domain reconstruction algorithms and numerical simulations for thermoacoustic tomography in various geometries," IEEE Trans. Biomed. Eng., vol. 50, no. 9, pp. 1086-1099, Sep. 2003.

[8] S. Norton, "Reconstruction of a two-dimensional reflecting medium over a circular domain: exact solution," J. Acoust. Soc. Am., vol. 67, no. 4, pp. 1266-1273, 1980.

[9] S. Norton and M. Linzer, "Ultrasonic reflectivity imaging in three dimensions: exact inverse scattering solutions for plane, cylindrical, and spherical apertures," IEEE Trans. Biomed. Eng., vol. BME-28, pp. 202-220, 1981.

[10] D. Finch, S. Patch, and S. Rakesh, "Determining a function from its mean values over a family of spheres," SIAM J. Math. Anal., vol. 35, pp. 1213-1240, 2004.

[11] X. Pan, Y. Zou, and M. A. Anastasio, "Data redundancy and reduced-scan reconstruction in reflectivity tomography," IEEE Trans. Image Process., vol. 12, no. 7, pp. 784-795, Jul. 2003.

[12] M. Anastasio, Y. Zou, and X. Pan, "Reflectivity tomography using temporally truncated data," in Proc. IEEE EMBS/BMES Conf., vol. 2, 2002, pp. 921-922.

[13] Y. Zou, X. Pan, and M. Anastasio, "Data truncation and the exterior reconstruction problem in reflection-mode tomography," in IEEE Nuclear Science Symp. Conf. Rec., vol. 2, 2002, pp. 726-730.

[14] E. Y. Sidky and X. Pan, "Image reconstruction with a half-detector in single-photon emission computed tomography with nonuniform attenuation," Opt. Eng., vol. 42, pp. 2506-2513, 2003.

[15] G. Paltauf, J. Viator, S. Prahl, and S. Jacques, "Iterative reconstruction algorithm for optoacoustic imaging," J. Acoust. Soc. Am., vol. 112, pp. 1536-1544, 2002.

[16] Y. Zhulina, "Optimal statistical approach to optoacoustic image reconstruction," Appl. Opt., vol. 39, pp. 5971-5977, 2000.

[17] Y. Vardi and D. Lee, "From image deblurring to optimal investments: maximum likelihood solutions for positive linear inverse problems," $J$. Roy. Statist. Soc. B, vol. 55, pp. 569-612, 1993.

[18] H. Barrett, D. Wilson, and B. Tsui, "Noise properties of the EM algorithm I. Theory," Phys. Med. Biol., vol. 39, pp. 833-846, 1994.

[19] J. Qi, "A unified noise analysis for iterative image estimation," Phys. Med. Biol., vol. 48, pp. 3505-3519, 2003.

[20] X. Pan and C. E. Metz, "Analysis of noise properties of a class of exact methods of inverting the 2D exponential Radon transform," IEEE Trans. Med. Imag., vol. 14, no. 4, pp. 659-668, Dec. 1995.

[21] G. Ku, X. Wang, G. Stoica, and L. V. Wang, "Multiple-bandwidth photoacoustic tomography," Phys. Med. Biol., vol. 49, pp. 1329-1338, 2004. 